\title{
PARÁMETROS PARA EL MANEJO DEL AGUA EN TOMATE Y CHILE DULCE HIDROPÓNICO BAJO INVERNADERO
}

\begin{abstract}
Palabras clave: Manejo del riego; potencial hídrico; humedad del sustrato; intervalo hídrico óptimo; punto de relleno.
\end{abstract}

Keywords: Irrigation management; water potential; substrate moisture; optimal water content interval; refill point.

Recibido: 09/10/17

RESUMEN

El intervalo hídrico óptimo, basado en umbrales de humedad volumétrica en el sustrato $\left(\theta_{\mathrm{vs}}\right)$, permite un control preciso del volumen y la frecuencia de riego. Se determinaron los umbrales de $\theta_{\mathrm{vs}}$ para el manejo del riego en los cultivos de tomate y chile dulce en fibra de coco en los invernaderos de la Estación Experimental Agrícola Fabio Baudrit Moreno (EEAFBM), Alajuela, Costa Rica, en el 2015. En ambos cultivos se aplicó un tratamiento con riego (CR) y otro sin riego (SR), en un diseño completamente aleatorio. Se evaluó el contenido de $\theta_{\mathrm{vs}}$, el potencial hídrico $\left(\psi_{\mathrm{h}}\right)$ y la temperatura de la hoja $\left(\mathrm{T}_{\mathrm{h}}\right)$ y se estimó la evapotranspiración de ambos cultivos mediante un balance de agua en el sustrato. El umbral de $\theta_{\text {vs }}$ en la fibra de coco, para el manejo del riego en ambos cultivos, podría establecerse en el rango de agua fácilmente disponible de $56 \%$ a $38 \%$, donde los valores de $\psi_{\mathrm{h}}$ fueron inferiores al umbral crítico de $<-1$ MPa reportado por diferentes autores y las $T_{h}$ fueron similares a la temperatura del aire. En los tratamientos CR, los promedios de $\theta_{\mathrm{vs}}$ en tomate $(44 \%)$ y chile dulce $(50 \%)$ estuvieron dentro del rango de agua fácilmente disponible $(38 \%$ a $56 \%$ ) de la fibra

* Universidad de Costa Rica, Estación Experimental Agrícola Fabio Baudrit Moreno, Facultad de
Aceptado: 05/02/18

\begin{abstract}
Water management parameters in hydroponic tomatoes and sweet pepper under greenhouse. The optimal water interval based on volumetric moisture thresholds in the substrate $\left(\theta_{\mathrm{vs}}\right)$, allows precise control of the volume and frequency of irrigation. The research determined the thresholds of $\theta_{\mathrm{vs}}$ for irrigation management in tomato and sweet pepper crops in coconut fiber in the greenhouses at the Fabio Baudrit Moreno Agricultural Research Station (EEAFBM), Alajuela, Costa Rica, 2015. In both crops, there were 2 treatments: with irrigation (CR) and without irrigation (SR), in a completely random design. The content of water $\theta_{\mathrm{vs}}$, the water potential $\left(\psi_{h}\right)$ and the leaf temperature $\left(T_{h}\right)$ were evaluated and the evapotranspiration of both crops was estimated by means of a water balance in the substrate. The $\theta_{\mathrm{vs}}$ threshold for water management in coconut fiber and both crops could be established in the readily available water range of $56 \%$ to $38 \%$, where the $\psi_{\mathrm{h}}$ values were below the critical threshold of $<-1 \mathrm{MPa}$ reported by different authors and the $T_{h}$ were similar to the air temperature. The averages of $\theta_{v s}$ in tomato (44\%) and sweet pepper (50\%) in CR treatments
\end{abstract}


de coco. En las pruebas SR de ambos cultivos, la $\theta_{\mathrm{vs}}$ descendió a un valor promedio de $32 \%$, próximo al punto de marchitez permanente. En $\psi_{\mathrm{h}} \mathrm{y} \mathrm{T}_{\mathrm{h}}$ las diferencias entre experimentos fueron de mayor magnitud al m.d. del día 2, donde los tratamientos CR fueron -0,84 MPa en tomate y -0,98 MPa en chile dulce; mientras que en las prácticas SR fueron -1,24 $\mathrm{MPa}$ en tomate y -1,3 $\mathrm{MPa}$ en chile dulce. Al m.d. del día 2, las $\mathrm{T}_{\mathrm{h}}$ de ambos cultivos en los diseños SR y CR fueron similares e inferiores, respectivamente, a la temperatura del aire $\left(32,9^{\circ} \mathrm{C}\right)$.

\section{INTRODUCCIÓN}

Actualmente, los temas de mayor importancia global son el cambio climático, la migración y la crisis del agua (WWAP 2016). Al mismo tiempo, el crecimiento demográfico mundial proyectado en 9000 millones de habitantes para el año 2050 (Tilman et al. 2002) supone un incremento sustancial en la demanda mundial de alimentos y, consecuentemente, en la demanda de agua para su producción. La escasez de agua dulce para riego podría duplicar los efectos perjudiciales del cambio climático en la agricultura, debido a la reducción de los rendimientos de cultivos básicos para la alimentación mundial como son maíz, soja, trigo y arroz (Elliott et al. 2014).

Lo anterior plantea la necesidad de realizar un uso eficiente del agua dulce disponible en el planeta. Diferentes criterios asociados a la interacción medio de cultivo-planta-atmósfera han sido utilizados mediante monitorización en tiempo real para el manejo de precisión del riego (Calado et al. 1990 citado por López et al. 2009.

Los métodos asociados al clima, tales como Penman Monteith, consideran parámetros climáticos tales como la radiación solar, temperatura, humedad del aire y velocidad del viento (Allen et al. 2006), sin embargo, su uso está were within the range of readily available water (38\% to 56\%) of the coconut fiber. In the SR treatments of both crops, the $\theta_{\mathrm{vs}}$ decreased to an average value of $32 \%$, close to the permanent wilting point. The differences between treatments of the $\psi_{\mathrm{h}}$ and $\mathrm{T}_{\mathrm{h}}$ were of higher magnitude at midday of $2^{\text {nd }}$ day. The $\psi_{\mathrm{h}}$ in CR treatments were $-0.84 \mathrm{MPa}$ and $-0.98 \mathrm{MPa}$, meanwhile in SR treatments they were $-1.24 \mathrm{MPa}$ and $-1.3 \mathrm{MPa}$, in tomato and sweet pepper respectively. At midday of $2^{\text {nd }}$ day, the $T_{h}$ of both crops and treatments were similar and lower, respectively, to the air temperature $\left(32.9^{\circ} \mathrm{C}\right)$.

limitado por la falta de datos disponibles en algunas regiones del mundo.

Dentro de los parámetros de planta están el $\psi_{\mathrm{h}}$ y la temperatura de la hoja $\left(\mathrm{T}_{\mathrm{h}}\right)$ (Ben-Asher et al. 1992), la contracción del tallo (Corell et al. 2014), el contenido relativo de agua y la conductancia estomática (García-Petillo 2008). El $\psi_{\mathrm{h}}$ ha sido utilizado principalmente con enfoques fisiológicos y ha tenido menos aplicación a nivel comercial, ya que el proceso de medición es lento y poco práctico (Ben-Asher et al. 1992).

El uso de parámetros asociados al medio de cultivo incluye temperatura, conductividad eléctrica, concentración de oxígeno y estado hídrico del medio. El uso de valores umbrales de potencial mátrico y contenido volumétrico de agua, como criterios para programar el volumen y frecuencia de riego, han sido descritos como intervalo de riego, porcentaje de agotamiento permisible, punto de relleno, umbrales de riego, límite superior e inferior de riego, entre otros (Thompson et al. 2007a y 2007b, Ali 2010, Van Der Westhuizen 2009, Incrocci et al. 2014, Pardossi et al. 2009, Pardossi y Incrocci 2011, van Iersel et al. 2010).

El límite superior es el valor máximo de contenido volumétrico de agua o potencial 
mátrico por encima del cual hay un exceso de aplicación de agua y usualmente corresponde a la capacidad de contenedor. El límite inferior es el valor mínimo a partir del cual el cultivo entra en estrés hídrico, lo que indica cuándo regar. El intervalo entre los límites superior e inferior permite determinar el volumen de riego y con ello la automatización y control del riego en tiempo real mediante sensores (Thompson et al. 2007a y 2007b, Ali 2010, Van Der Westhuizen 2009, Incrocci et al. 2014, Pardossi et al. 2009, Pardossi y Incrocci 2011, van Iersel et al. 2010).

Los valores umbrales de potencial mátrico y $\theta_{\mathrm{vs}}$ reportados en la literatura son variables, ya que dependen de diferentes factores tales como el genotipo, el clima y manejo agronómico, el sustrato, morfología del contenedor y la técnica de cultivo, entre otros.

La mayoría de estudios han sido realizados en cultivos en suelo a campo abierto, muy pocos en sustrato bajo invernadero, enfocados en evaluar el efecto del agotamiento hídrico sobre el rendimiento y la calidad del producto, sin relacionarlo con el estado hídrico de la planta y con el clima.

El $\psi_{\mathrm{h}}$ de la planta es un excelente indicador del estrés hídrico de las plantas, ya que gobierna el flujo hídrico en el continuo sueloplanta-atmósfera. Algunos estudios han demostrado que el estrés hídrico a nivel de planta puede alcanzarse mucho antes de conseguir un determinado nivel de agotamiento de $\theta_{\mathrm{vs}}$ (Thompson et al. 2007a y 2007b).

El tipo de sustrato, su granulometría y la forma del contenedor, podrían afectar el uso de umbrales de $\theta_{\text {vs }}$ para el manejo del riego, ya que influyen en el volumen y la frecuencia de este (Arguedas et al. 2006). Por tanto, es importante determinar las características físicas del sustrato, puesto que existe alta variabilidad según su origen y naturaleza (Abad et al. 2002). La relación entre contenido volumétrico de agua y el potencial matricial del sustrato, permite establecer el rango de humedad en el cual el agua es fácilmente disponible para la planta (Vence et al. 2013). Arguedas et al. (2006) determinaron que a mayor altura del contenedor, menor volumen de agua disponible y que materiales como peat moss requirió riegos cortos y más frecuentes que la grava.

Chen et al. (2013) y Kuşçu et al. (2014) demostraron que la etapa de floración-fructificación-cosecha del cultivo es la de mayor sensibilidad a condiciones de estrés hídrico, lo cual influyó significativamente sobre el rendimiento y la calidad. La etapa de crecimiento vegetativo es relativamente corta, con un incremento variable y continuo de biomasa vegetativa que se estabiliza hasta que inicia el crecimiento de frutos. Por el contrario, la etapa productiva es más larga y coinciden simultáneamente ciclos de crecimiento vegetativo, floración, formación y crecimiento de frutos (Gallardo et al. 2011). Kusçu et al. (2014) demostraron que en la etapa productiva de tomate, el manejo de niveles de agotamiento de $\theta_{\text {vs }}$, sin afectar el estado hídrico de la planta, contribuyó a incrementar hasta un $42 \%$ la eficiencia de uso de agua sin afectar el rendimiento y la calidad de frutos.

Por tanto, el uso de valores predefinidos pueden ser imprecisos en diferentes condiciones de cultivo, al plantearse la necesidad de realizar estos estudios in situ, al considerarse la interacción entre genotipo, el clima y el manejo agronómico, y al asociarlos con indicadores de estrés hídrico en la planta. El objetivo de este estudio fue identificar valores umbrales de humedad en el sustrato, como criterio para el manejo del riego durante la etapa de producción de cultivos de chile dulce y tomate en fibra de coco bajo invernadero.

\section{MATERIALES Y MÉTODOS}

El estudio se realizó en la Estación Experimental Fabio Baudrit Moreno (EEFBM), ubicada en San José de Alajuela, Costa Rica $\left(10^{\circ} 01^{\prime} \mathrm{N}\right.$ y $84^{\circ} 16^{\prime} \mathrm{W}, 840 \mathrm{msnm}$ ), con valores promedios de precipitación anual, temperatura y humedad relativa de $1940 \mathrm{~mm}, 22^{\circ} \mathrm{C}$ y $78 \%$, respectivamente. Se utilizó un invernadero multitúnel de hierro galvanizado, con cubierta de polietileno 
transparente $(200 \mu \mathrm{m})$ y malla anti-insectos (43 mesh) en las paredes, formado por 4 módulos de $9,75 \mathrm{~m}$ de ancho y $50 \mathrm{~m}$ de largo, alturas de $6 \mathrm{~m}$ al centro del túnel y $4 \mathrm{~m}$ a la canoa.

Durante la época de lluvia del 2015, se realizaron simultáneamente 2 cultivos hidropónicos, uno de tomate Cherry (Solanum lycopersicum L.) y otro de chile dulce "bell pepper" (Capsicum апnиит). En tomate se utilizó el cultivar Super Suncherry, de crecimiento indeterminado, con ramos de frutos con un peso promedio de 20-30 $\mathrm{g}$ de color rojo intenso, un potencial de producción de 150-180 t.ha-1 y con buena tolerancia a enfermedades. En chile dulce se utilizó el híbrido Polaris, el cual presenta plantas vigorosas, frutos uniformes de color amarillo con un peso de $160 \mathrm{a}$ $185 \mathrm{~g}$, se adapta muy bien a temperaturas de $20 \mathrm{a}$ $30^{\circ} \mathrm{C}$, con resistencia al virus $\mathrm{Y}$ de la papa (PVY) y alta resistencia a la manipulación pos-cosecha.

Durante el experimento los promedios globales de temperatura y humedad relativa, máximas, mínimas y promedios, fueron $23,7^{\circ} \mathrm{C}$, $15,5^{\circ} \mathrm{C}$ y $19,5^{\circ} \mathrm{C}$ y $83 \%, 45 \%$ y $65 \%$, en el mismo orden. El promedio máximo de radiación PAR incidente de $1293 \mu \mathrm{mol} \mathrm{m}{ }^{-2} \cdot \mathrm{s}^{-1}$ al mediodía.

En ambos cultivos se utilizó como sustrato fibra de coco en tablas de $3 \mathrm{~kg}$ en seco, con dimensiones de 16 × 3.5 x $100 \mathrm{~cm}$ y un volumen de $29 \mathrm{~L}$ una vez hidratada. A dicho sustrato se le determinó las características físicas utilizando métodos de laboratorio para sustratos (Ansorena 1994). Las tablas fueron hidratadas aplicando riegos cortos y frecuentes cada 20 minutos hasta saturación, los cuales alcanzaron un volumen aproximado de 28,8 L por tabla. Posteriormente, se realizaron 2 cortes en forma de $\mathrm{T}$ invertida de aproximadamente $4 \mathrm{~cm}$ en la parte inferior del saco de cultivo y lo más próximos al suelo para drenar el exceso de agua aplicada. Los sacos de cultivo fueron colocados consecutivamente, los cuales formaron hileras simples espaciadas a 1,5 m.

En cada cultivo el área experimental estuvo conformada por 6 hileras de 16 tablas con 3 plantas cada una, para un total de 288 plantas.cultivo $^{-1}$, a una densidad de 2 plantas $\mathrm{m}^{-2}$. En ambos cultivos la siembra se realizó por trasplante. En tomate se utilizaron plántulas de 28 días, con 4-5 hojas verdaderas, mientras que en chile dulce se utilizaron plántulas de 6 semanas de edad, con aproximadamente 4-5 hojas verdaderas.

La planta de tomate fue formada a 2 tallos, mientras que en chile dulce se formó a 4 tallos por planta, los cuales fueron sujetados con cuerdas de nylon a las líneas de alambre colocadas en la parte superior del cultivo. Además, durante el ciclo de ambos cultivos se realizaron podas de brotes laterales, deshojas, y podas de saneamiento y aireación por debajo del último fruto cosechado. El manejo fitosanitario de ambos cultivos fue similar al utilizado en cultivos comerciales bajo invernadero, de acuerdo con el monitoreo semanal de plagas.

Cada cultivo tenía un sistema de riego por goteo superficial independiente, con tuberías de polietileno paralelas a las hileras de cultivo y provistas de 4 goteros autocompensados y antidrenantes $\left(21 . h^{-1}\right)$ por tabla de cultivo. La nutrición mineral se realizó mediante la aplicación de soluciones nutritivas específicas para cada cultivo, según etapa fenológica (Cuadro 1). El pH de la solución nutritiva aplicada fue ajustado a un valor de 5,7 al utilizar ácido nítrico $\left(56 \% \mathrm{v} / \mathrm{v}, 167 \mathrm{~g} . \mathrm{l}^{-1}\right)$. 
Cuadro 1. Concentración de nutrientes $\left(\mathrm{mg} \cdot \mathrm{l}^{-1}\right)$ en solución nutritiva utilizada por etapa fenológica en el cultivo de tomate cherry y chile dulce "bell pepper". Alajuela, Costa Rica. Diciembre 2015.

\begin{tabular}{|c|c|c|c|c|c|c|c|c|c|c|c|c|c|}
\hline Cultivo* & $\mathrm{Ca}$ & $\mathrm{K}$ & $\mathrm{Mg}$ & $\mathrm{N}$ & $S$ & $\mathrm{P}$ & $\mathrm{Fe}$ & $\mathrm{Cu}$ & $\mathrm{Mn}$ & $\mathrm{Zn}$ & B & Mo & $\mathrm{CE}$ \\
\hline \multirow{4}{*}{$\begin{array}{l}\stackrel{0}{\tilde{\Xi}} \\
\stackrel{\Xi}{0} \\
\stackrel{0}{0}\end{array}$} & 140 & 180 & 50 & 120 & 84 & 50 & 1,0 & 0,10 & 0,50 & 0,10 & 0,51 & 0,06 & 1,5 \\
\hline & 160 & 250 & 50 & 135 & 96 & 50 & 1,50 & 0,15 & 0,75 & 0,15 & 0,50 & 0,05 & 1,9 \\
\hline & 170 & 300 & 50 & 150 & 100 & 50 & 2,5 & 0,15 & 1,25 & 0,15 & 0,50 & 0,05 & 2,0 \\
\hline & 170 & 280 & 40 & 170 & 110 & 45 & 2,0 & 0,23 & 1,0 & 0,23 & 0,50 & 0,08 & 2,3 \\
\hline \multirow{4}{*}{ 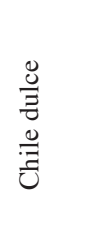 } & 140 & 182 & 37 & 130 & 65 & 40 & 1,0 & 0,23 & 0,80 & 0,23 & 0,50 & 0,08 & 1,4 \\
\hline & 150 & 205 & 45 & 140 & 74,95 & 46,9 & 1,50 & 0,23 & 0,80 & 0,23 & 0,50 & 0,08 & 1,6 \\
\hline & 172 & 260 & 50 & 152 & 105 & 46 & 1,60 & 0,23 & 0,80 & 0,23 & 0,51 & 0,08 & 2,0 \\
\hline & 195 & 300 & 50 & 169 & 119 & 50 & 2,0 & 0,23 & 1,0 & 0,23 & 0,50 & 0,08 & 2,3 \\
\hline
\end{tabular}

*Etapas de cultivo: 1: establecimiento, 2: desarrollo vegetativo y radicular, 3: crecimiento- inicio producción y 4: inicio producción en adelante.

El estudio se realizó durante la etapa de producción, en la cual coinciden simultáneamente ciclos de crecimiento vegetativo, floración, cuaje, llenado y maduración de frutos. Según estudios realizados por Chen et al. (2013) y Kuşçu et al. (2014), en dicha etapa el estrés hídrico tiene el mayor impacto negativo sobre el rendimiento y la calidad de frutos. En ambos cultivos se utilizaron 2 tratamientos, un control con riego (CR) y otro sin riego (SR). Un día antes ambos cultivos fueron regados abundantemente hasta obtener suficiente drenaje. En los tratamientos CR de ambos cultivos el riego se programó cada hora, desde 7 a.m. a 5 p.m, mediante un programador (timer) y fue ajustado según el objetivo de obtener un drenaje de entre $15 \%$ y $20 \%$. En los tratamientos SR de ambos cultivos, después de regar abundantemente, cesar el drenaje y alcanzar la capacidad de contenedor en el sustrato, se suspendió definitivamente la aplicación de agua de riego.

Los 2 tratamientos fueron distribuidos en un diseño completamente aleatorio con 4 repeticiones y 8 unidades experimentales (UE) por cultivo. La UE consistió en 3 hileras conformadas por 4 tablas de cultivo con 3 plantas cada una, para un total de 12 tablas y y 36 plantas por
UE. En cada repetición la unidad de muestreo (UM) consistió en 2 tablas centrales de la hilera central. Las hileras laterales y plantas ubicadas al final de la hilera central se utilizaron como plantas de borde.

Las variables evaluadas fueron $\theta_{\mathrm{vs}}, \psi_{\mathrm{h}} \mathrm{y}$ $\mathrm{T}_{\mathrm{h}}$ en la planta, durante un periodo de 7 días, hasta que las plantas del tratamiento SR colapsaron, debido a la falta de agua. La $\theta_{\mathrm{vs}}$ se midió 6 veces por día cada 2 horas entre 6 a.m. y 4 p.m. El $\psi_{h}$ y la $T_{h}$ se midieron 2 veces diariamente, antes del amanecer (a.m.) y al medio día (m.d.). La $\mathrm{T}_{\mathrm{h}}$ se midió en 3 hojas jóvenes por repetición, completamente desarrolladas y expuestas a la radiación solar, utilizando un termómetro infrarrojo (Cooper-Atkins $\left.{ }^{\circledR} \bmod .412\right)$ a una distancia de $40 \mathrm{~cm}$ en dirección perpendicular a la hoja. El $\psi_{\mathrm{h}}$ se determinó con la cámara de Scholander (Soil Moisture Equipment Corp ${ }^{\circledR}$. mod. 3005) en 3 hojas jóvenes por repetición, expuestas al sol, que se colocaron en una bolsa plástica y se envolvieron con papel de aluminio para que el $\Psi_{\mathrm{h}}$ se equilibrara con el $\Psi$ xilemático o del brote (McCutchan y Shackel, 1992, citados por Ferreyra et al. 2007). Se extrajo la hoja de la planta, se colocó en una hielera e inmediatamente se 
transportó al laboratorio para medir el $\Psi_{\mathrm{h}}$ según Ferreyra et al. (2007).

En los tratamientos CR de ambos cultivos, se realizó un balance de agua diario en el sistema sustrato, en el cual se consideraron como entradas el riego aportado (R), y como salidas la evapotranspiración del cultivo $\left(\mathrm{ET}_{\mathrm{c}}\right)$, el drenaje (D) y la variación de humedad en el sustrato $\left(\Delta \theta_{\mathrm{vs}}\right)$. El volumen de riego diario $(\mathrm{R})$ fue estimado a partir del caudal promedio de los goteros, el número de goteros por planta y el tiempo de riego. El caudal promedio de los goteros fue obtenido mediante el coeficiente de uniformidad del sistema de riego (Christiansen 1942).

El volumen de drenaje (L) en los tratamientos CR, se midió diariamente a las 6 a.m. antes de iniciar el primer riego del día, desde una bandeja de drenaje impermeabilizada con plástico, la cual contenía una tabla de cultivo con 3 plantas. En un extremo de la bandeja se colocó una manguera plástica para recolectar el agua drenada. La bandeja se cubrió con plástico plata/negro para evitar la evaporación del agua drenada.

La $\theta_{\text {vs }}$ fue medida en la parte central del perfil del sustrato en la tabla de cultivo, en el que se utilizó un sensor TDR (Time Domain Reflectometry, model GS3 Decagon Devices) antes del inicio de los riegos diarios. La $\Delta \theta_{\mathrm{vs}}$ fue obtenida por diferencia entre los contenidos de humedad volumétrica a las 6 a.m. del día actual $\left(\theta_{\text {vsa }}\right)$ y la 6 a.m. del día posterior $\left(\theta_{v s f}\right)$. Todos los componentes del balance fueron expresados en 1.planta ${ }^{-1}$. día ${ }^{-1}$ y para estimar la evapotranspiración diaria del cultivo $\left(\mathrm{ET}_{\mathrm{c}}\right)$ se utilizó la siguiente ecuación, a partir de los componentes del balance de agua en el sustrato descrito por Soto et al. 2014.

$$
\mathrm{ET}_{\mathrm{c}}=\mathrm{R}-\mathrm{D}-\left(\Delta \theta_{\mathrm{i}}-\Delta \theta_{\mathrm{f}}\right)(\text { Ecuación } 1)
$$

En los cultivos sin riego, al considerar que no hay entradas de agua mediante el riego y, por tanto, no hay agua drenada, se asume que la $\mathrm{ET}_{\mathrm{c}}$ diaria fue equivalente a la pérdida de agua en el sustrato $\left(\Delta \theta_{\mathrm{vs}}=\theta_{\mathrm{vsa}}-\theta_{\mathrm{vsf}}\right)$.

Para evaluar los efectos de los tratamientos (T), del número de días (D) y de la hora del día $(\mathrm{H})$, se consideraron todos los datos medidos cada 2 horas (6 a.m. a 4 p.m.) durante los 7 días, mediante el análisis de los datos con el siguiente modelo estadístico mixto de mediciones repetidas en el tiempo.

Yijkl= M+ Ti+ Rij+ Dk + TDik +TDRijk+ Hl + THijl+THRijl+ TDHikl + Eijkl; en el cual los términos de error utilizados en las pruebas estadísticas fueron los siguientes: para probar el efecto Ti se empleó Rij; para probar Dk y TDik se empleó TDRijk; para probar los efectos $\mathrm{Hl}$ y THil se empleó THRijl y para probar la triple interacción TDHikl se usó el término de error del modelo Eijkl. Lo anterior, de conformidad con la regla para determinar las pruebas apropiadas de F, según la cual, para probar un efecto mediante una prueba estadística de $\mathrm{F}$, el cuadrado medio del error (denominador) debe ser aquel cuyo valor esperado es idéntico al valor esperado del cuadrado medio del efecto a probar (numerador), excepto que carece del término correspondiente al efecto a probar (Zar 1999, Steel y Torrie 1980). Para el análisis de la información generada se utilizó el paquete estadístico SAS versión 9.3 (SAS Institute Inc., Cary, NC, USA.).

\section{RESULTADOS}

\section{Humedad volumétrica y evapotranspiración}

En la curva característica de desorción de agua de la fibra de coco, la relación entre la $\theta_{\mathrm{vs}} \mathrm{y}$ la tensión de succión matricial del sustrato fueron $56 \%$ a $1,0 \mathrm{kPa}$ (capacidad de contenedor: $\mathrm{CC}$ ), $44 \%$ a $2,5 \mathrm{kPa}, 38 \%$ a $5 \mathrm{kPa}$ y $30 \%$ a $10 \mathrm{kPa}$ (punto de marchitez permanente: PMP) (Figura 1a). 

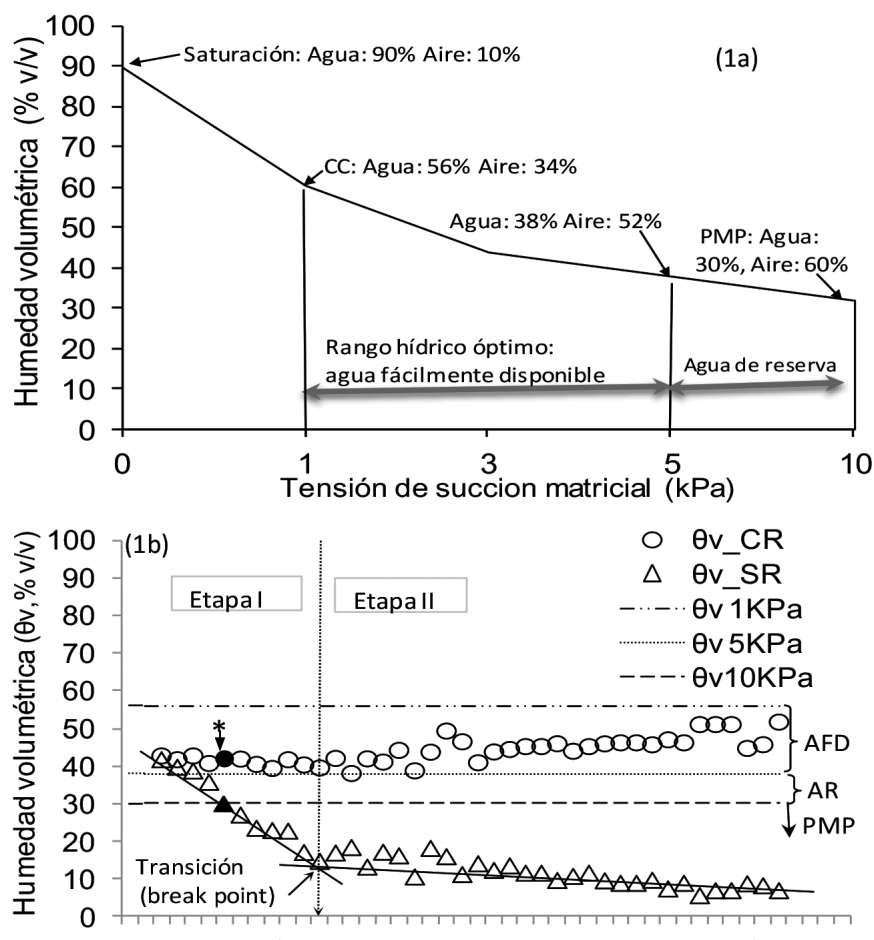

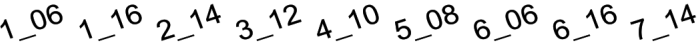

Día_hora

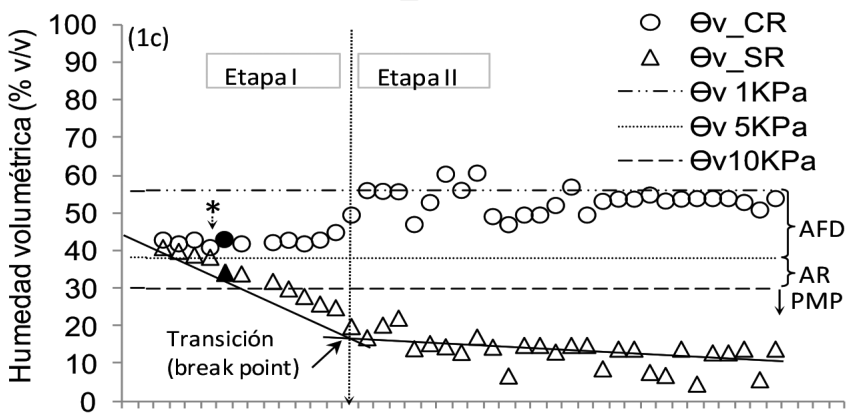

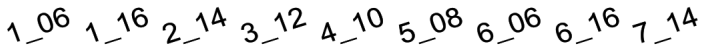

Día_hora

Fig. 1. Curva característica de desorción de agua de la fibra de coco (1a) y evolución de la humedad volumétrica en sustrato $\left(\theta_{\mathrm{vs}}\right)$ según día y hora en los cultivos de tomate (1b) y chile dulce (1c) bajo tratamientos con riego (CR) y sin riego (SR). El símbolo * indica el día_hora a partir del cual inician diferencias estadísticamente significativas $(\mathrm{p}<0,05)$ entre tratamientos. Las siglas AFD, AR y PMP significan el agua fácilmente disponible, agua de reserva y punto de marchitez permanente en la fibra de coco, respectivamente.

En el cultivo de tomate $\mathrm{CR}$, la $\theta_{\mathrm{vs}}$ fluctuó entre $42 \%$ y $51 \%$, con un promedio de $44 \%$ durante el ciclo (Figura 1b). El tratamiento SR mostró una primer etapa, donde ocurre una rápida reducción de la $\theta_{\text {vs }}$ de entre los días 1 y 3 , y una segunda etapa, donde las pérdidas de humedad fueron de menor magnitud (Figura 1b). Durante la etapa I, la $\theta_{\mathrm{vs}}$ disminuye progresivamente desde 
42 hasta un $15 \%$ a las 12 m.d. del día 3, en la cual presentó diferencias $(\mathrm{p}<0,05)$ entre tratamientos a las 6 horas del día 2 (Figura 1b). En la etapa II, la $\theta_{\mathrm{vs}}$ disminuye ligeramente con pequeñas variaciones hasta alcanzar un valor de $7 \%$ al final del estudio.

En chile dulce $\mathrm{CR}$, la $\theta_{\mathrm{vs}}$ estuvo en un rango de $42 \%$ a $60 \%$ y un promedio de $50 \%$ (Figura 1c). En el tratamiento SR la $\theta_{\text {vs }}$ disminuyó paulatinamente desde $42 \%$ hasta $5 \%$ el día 7 . En una primer etapa, la $\theta_{\mathrm{vs}}$ disminuyó rápidamente hasta un $17 \%$ a las 12 horas del día 3, en la que presentó diferencias $(\mathrm{p}<0,05)$ respecto a $\mathrm{CR}$ a partir de 6 horas del día 2. En la etapa II, la pérdida de $\theta_{\mathrm{vs}}$ es de menor magnitud, con una tendencia a estabilizarse y con un promedio de 14\% (Figura 1c).

\section{Efecto sobre la evapotranspiración del cultivo} $\left(\mathbf{E T}_{\mathrm{c}}\right)$

En los cultivos de tomate y chile dulce la evolución de la $\theta_{\mathrm{vs}}$ (Figura 1b, 1c) fue consecuente con el comportamiento mostrado en la evolución de la $\mathrm{ET}_{\mathrm{c}}$ de los tratamientos CR y SR, y Pemman-Montieh FAO-56 (Allen et al. 2006) (Figura 2b, 2c). 

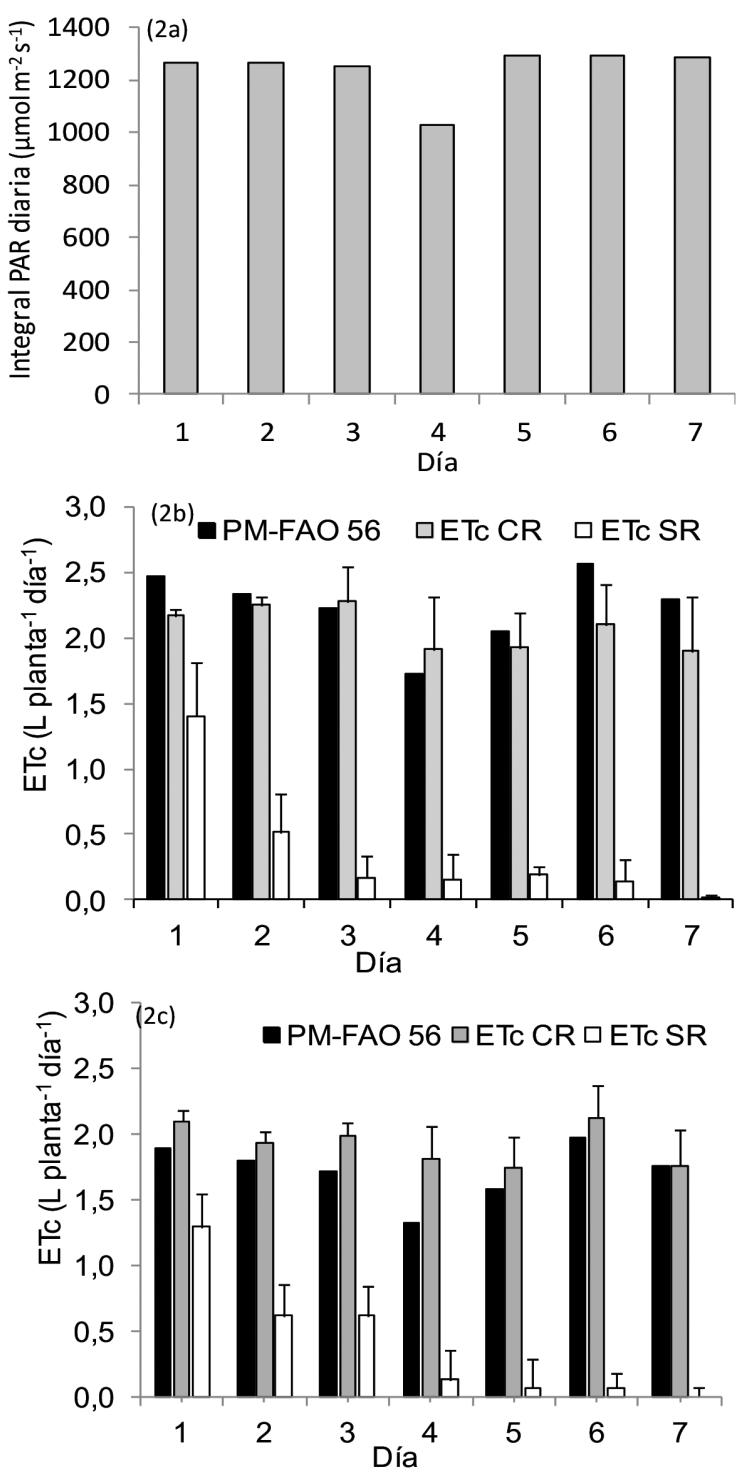

Fig. 2. Evolución diaria de la integral de radiación solar fotosintéticamente activa (PAR) (2a), y evapotranspiración de los cultivos (ET) de tomate (2b) y chile dulce (2c) con riego (CR), sin riego (SR) y estimada según Pemman-Montieh FAO-56 (PM-FAO 56).

$\mathrm{La} \mathrm{ET}_{\mathrm{c}}$ de los cultivos de tomate y chile dulce CR también fue consecuente con la evolución diaria de la integral de radiación fotosintéticamente activa (PAR), puesto que mostró los menores valores de PAR (Figura 2a) y de $\mathrm{ET}_{\mathrm{c}}$ el día 4. La ET cluctuó entre 1,9 a 2,3 1.planta-1 . día $^{-1}$ en tomate (Figura 2b) y entre 1,75 hasta 2,1 1.planta ${ }^{-1} \cdot$ día $^{-1}$ en chile dulce (Figura 2c). La ET de ambos cultivos CR fue similar a la de PMFAO-56, con una diferencia promedio durante todo el periodo de $7,83 \%$ para tomate y de $10 \%$ en chile dulce. 
En los cultivos de tomate y en chile dulce, el tratamiento SR mostró una reducción apreciable en la $\mathrm{ET}_{\mathrm{c}}$ los primeros 2 días, luego se mantuvo en valores muy bajos hasta el final del periodo (Figura 2b). $\mathrm{La} \mathrm{ET}_{\mathrm{c}}$ del tratamiento SR, comparada con el tratamiento CR, disminuyó un $35 \%$ el día 1, un $77 \%$ el día 2 , hasta alcanzar un 99\% del día 7, con un máximo de 1,4 1.planta ${ }^{-1}$ el día 1. En chile dulce, la $\mathrm{ET}_{\mathrm{c}}$ del tratamiento SR respecto a CR, disminuyó un $38 \%$ el día 1 , un 68,2\% el día 2, hasta un 100\% el día 7 (Figura 2c), con 1,3 1.planta ${ }^{-1}$ el día 1 .

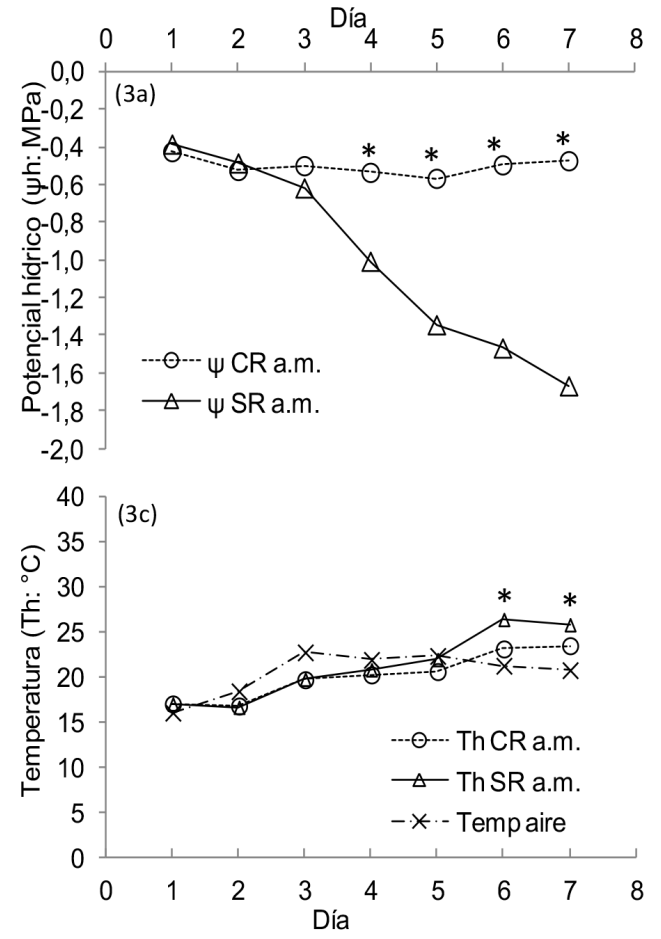

\section{Parámetros de planta}

En ambos cultivos de tomate y chile dulce CR y SR, el comportamiento en la evolución del $\psi_{\mathrm{h}}$ antes del amanecer (a.m.) y al medio día (m.d.) fue consecuente con el de la $\mathrm{T}_{\mathrm{h}}$ (Figura 3, Figura 4). Los valores de $\psi_{\mathrm{h}}$ a.m. en tomate CR oscilaron entre $-0,43 \mathrm{MPa}$ hasta $-0,57 \mathrm{MPa}$, mientras que en el tratamiento SR variaron de $-0,39 \mathrm{MPa}$ hasta $-1,67 \mathrm{MPa}$ (Figura 3a), en el que presentaron diferencias $(p<0,05)$ a partir del día 4 , donde el tratamiento SR fue -0,47 MPa inferior al CR. Las mayores diferencias se presentaron durante el m.d., donde a partir del día 2 el cultivo SR fue $-0,40$ MPa inferior $(\mathrm{p}<0,05)$ al cultivo CR (Figura $3 b)$.

Fig. 3. Evolución diaria del potencial hídrico (3a, 3b) y la temperatura de la hoja (3c, 3d), al amanecer (a.m.) y al medio día (m.d.) en el cultivo de tomate hidropónico bajo invernadero, bajo tratamientos con riego (CR) y sin riego (SR). El símbolo * indica diferencias estadísticamente significativas $(\mathrm{p}<0,05)$ entre tratamientos. 

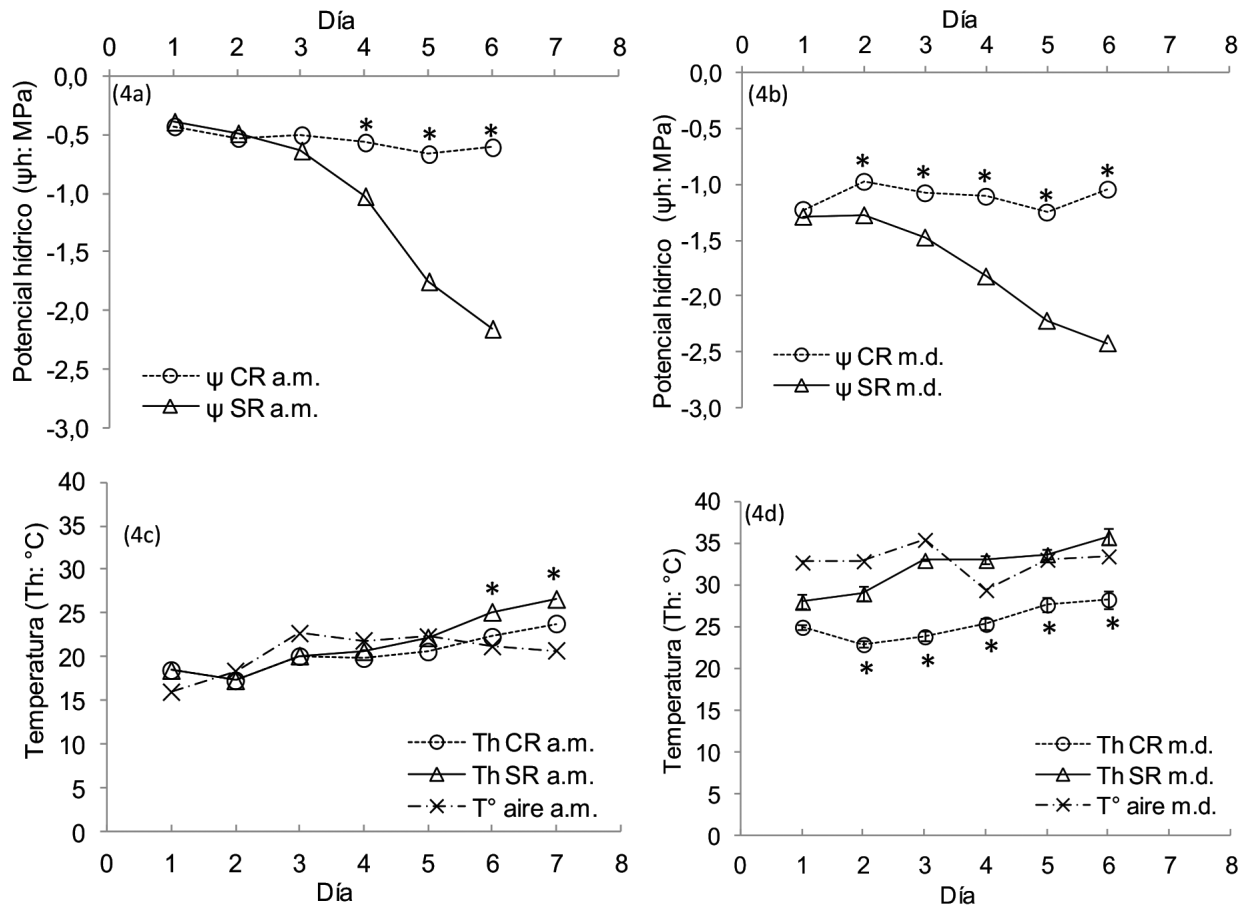

Fig. 4. Evolución diaria del potencial hídrico (4a, 4b) y la temperatura de la hoja (4c, 4d), al amanecer (a.m.) y al medio día (m.d.), en el cultivo de chile dulce hidropónico bajo invernadero, bajo tratamientos con riego (CR) y sin riego (SR). El símbolo * indica diferencias estadísticamente significativas $(\mathrm{p}<0,05)$ entre tratamientos.

En el cultivo de chile dulce CR los valores de $\psi_{\mathrm{h}}$ en a.m. oscilaron entre $-0,43 \mathrm{MPa}$ hasta $-0,66 \mathrm{MPa}$, mientras que en el cultivo SR fluctuaron desde -0,39 MPa hasta -2,16 MPa. Se presentaron diferencias entre tratamientos $(\mathrm{p}<0,05)$ a partir del día 4 a.m., donde el $\psi_{\mathrm{h}}$ del tratamiento SR fue -0,46 MPa inferior al cultivo CR (Figura 4a). El $\psi_{\mathrm{h}}$ al m.d. mostró diferencias $(\mathrm{p}<0,05)$ entre tratamientos del día 2 en adelante, donde el cultivo SR fue $-0,32 \mathrm{MPa}$ inferior que el cultivo CR (Figura 4b).

Coincidente con el comportamiento del $\psi_{\mathrm{h}}$, en ambos cultivos de tomate y chile dulce, las diferencias de $T_{h}$ entre tratamientos fueron menores en la mañana (Figura 3c, 4c) que al m.d. (Figura 3d, 4d). Las $\mathrm{T}_{\mathrm{h}}$ en a.m. fueron diferentes $(\mathrm{p}<0,05)$ a partir del día 6 en a.m. y a partir del día 2 en m.d. Dichas diferencias fluctuaron entre $3,3^{\circ} \mathrm{C}$ a las 6 a.m. en tomate (Figura $3 \mathrm{c}$ ) hasta $7,4^{\circ} \mathrm{C}$ en m.d. en chile dulce (Figura $4 \mathrm{~d}$ ).

\section{DISCUSIÓN}

En este estudio, coincidente con otros autores (Starr y Paltineanu 1998, Thompson et al. 2007a y 2007b, Dorji y Behboudianet 2005), los tratamientos SR de ambos cultivos presentaron un punto de inflexión que definió la transición del proceso de desorción de agua en el sustrato, desde i- una etapa de rápida pérdida de agua a ii- otra subsecuentemente más lenta (Figura 2a, 2b). Dicha transición se manifestó a las 12 horas del día 3 , cuando la $\theta_{\text {vs }}$ en tomate $(15 \%)$ y en chile dulce $(17 \%)$ fueron menores al punto de marchitez permanente $(30 \%)$ de la fibra de coco. 
La evolución de la $\theta_{\text {vs }}$ en los tratamientos $\mathrm{SR}$, coincidió con una reducción de la $\mathrm{ET}_{\mathrm{c}}$ (Figura 2) de $92,5 \%$ en tomate y de $67 \%$ en chile dulce, debido al agotamiento del agua fácilmente disponible en el sustrato. Consecuentemente, se reduce el $\psi_{\mathrm{h}}$ y se limita la capacidad de enfriamiento evaporatorio de la hoja incrementando la $\mathrm{T}_{\mathrm{h}}$ (BenAsher et al. 1992). La transición de etapas, según la $\theta_{\text {vs }}$, fue coincidente con en el comportamiento del $\psi_{\mathrm{h}} \mathrm{y}_{\mathrm{h}}$ en ambos cultivos SR, ya que a partir del día 3 al m.d. hubo una apreciable reducción en el $\psi_{\mathrm{h}}$ (Figura $4 \mathrm{a}, 4 \mathrm{~b}$ ) y consecuentemente un significativo incremento en la $T_{h}$, respecto a los tratamientos CR (Figura 4c, 4d).

La mayoría de estudios reportados en la literatura se han enfocado en evaluar el efecto del porcentaje de agotamiento de la humedad sobre el rendimiento y la calidad de fruto (Chen et al. 2013). Sin embargo, estudios realizados por Davies y Zhang (1991) y Tardieu y Davies (1992) citados por Kirda et al. (2004), demostraron que la regulación estomática es controlada a través de señales químicas enviadas desde las raíces hasta las hojas, lo cual explica porqué los umbrales de $\theta_{\mathrm{vs}}$ para ser utilizados como criterios de manejo del riego, deben ser determinados al relacionarlos con parámetros de planta. Por ello, en este estudio se evaluó paralelamente el $\psi_{\mathrm{h}}$ y la $\mathrm{T}_{\mathrm{h}}$, y se consideró la curva característica de desorción de agua de la fibra de coco (Figura 1a).

La disminución de la $\theta_{\mathrm{vs}}$ afecta la cantidad de agua fácilmente disponible para la planta, lo cual perjudica directamente la transpiración. En horas cercanas al amanecer, cuando el contenido de $\theta_{\mathrm{vs}}$ es cercano a capacidad de contenedor (CC) y las condiciones de clima son óptimas, los estomas se mantienen abiertos y la planta transpira a su potencial normal (Pilatti et al. 2012). La $\theta_{\mathrm{v}}$ en la fibra de coco de los cultivos de tomate y chile dulce CR estuvieron dentro del rango de agua fácilmente disponible para la planta, comprendido entre $56 \%(\mathrm{CC}$ a $-1 \mathrm{kPa})$ y $38 \%(-5 \mathrm{kPa})$, sin llegar a demandar el agua de reserva en el sustrato (Figura 1). Resultados similares en cultivos en sustratos han sido demostrados por Incrocci et al. (2014) y Van Der Westhuizen (2009).
Por otra parte, se presentaron diferencias en la $\theta_{\text {vs }}$ entre tratamientos $(\mathrm{p}<0,05)$ a las 6 horas del día 2 (Figura $1 \mathrm{~b}, 1 \mathrm{c}$ ), cuando los contenidos de la $\theta_{\text {vs }}$ fueron $32 \%$ en tomate y $34 \%$ en chile dulce. Dichos valores fueron similares al punto de marchitez permanente (PMP) de la fibra de coco (30\%: Figura 1). Al m.d. cuando la $\theta_{\mathrm{vs}}$ disminuyó cerca del PMP y las condiciones de clima eran críticas, probablemente la tasa de transpiración fue mayor que la de absorción. Consecuentemente, se redujo el $\psi_{\mathrm{h}}$, por lo que la planta cierra estomas como mecanismo de defensa para evitar el colapso. En dichas condiciones el cierre es el mecanismo más dominante, debido a su valor protector durante sequías (San Martín y Acevedo 2001).

El uso de umbrales de $\theta_{\mathrm{vs}}$ para el manejo del riego ha sido ampliamente descrito en cultivos en suelo (Thompson et al. 2007a y 2007b, Pilatti et al. 2012, Pardossi et al. 2009, Pardossi y Incrocci 2011, Capraro et al. 2008) y en menor escala en sustrato (van Iersel et al. 2010, Van Der Westhuizen 2009, Incrocci et al. 2014). Además, han sido utilizados para el control automático del riego mediante monitorización en tiempo real con sensores de $\theta_{\mathrm{vs}}$ en suelo (Navarro-Hellín et al. 2015, Vellidis et al. 2008, Goumopoulos et al. 2014, Coates et al. 2013) y en sustratos (Van Der Westhuizen 2009, Rhie y Kim 2017).

En este estudio, el comportamiento del $\psi_{\mathrm{h}}$ y la $\mathrm{T}_{\mathrm{h}}$ de ambos cultivos de tomate y chile dulce fue diferente al amanecer (a.m) respecto al m.d., lo cual coincide con estudios realizados por Dorji y Behboudian (2005). Al amanecer (a.m.), las diferencias entre tratamientos se presentaron el día 3 en $\psi_{\mathrm{h}}$ y el día 6 en $\mathrm{T}_{\mathrm{h}}(3 \mathrm{a}, 3 \mathrm{c}, 4 \mathrm{a}, 4 \mathrm{c})$. Por el contrario, al m.d. tanto el $\psi_{\mathrm{h}}$ como la $\mathrm{T}_{\mathrm{h}}$, presentaron diferencias $(\mathrm{p}<0,05)$ entre tratamientos el día 2 (Figura 3b, 3d, 4b, 4d).

Lo anterior se debe a que el valor del $\psi_{\mathrm{h}}$ depende del balance entre ganancia y pérdida de agua a través de la transpiración, que a su vez depende de las condiciones atmosféricas (Ali 2010). En horas a.m., la transpiración es menor que la absorción de agua, lo que incrementó el $\psi_{\mathrm{h}}$ y redujo la $\mathrm{T}_{\mathrm{h}}$, debido al descenso en la 
temperatura y al incremento de la humedad relativa. Por tanto, en a.m. el $\psi_{\mathrm{h}}$ representa el equilibrio hídrico entre sustrato-planta-atmósfera, lo cual redujo las necesidades de agua al mínimo. Consecuentemente, el $\psi_{\mathrm{h}}$ en condiciones a.m. es poco sensible al contenido de $\theta_{\mathrm{vs}}$, por lo que es un criterio poco aplicable al manejo del riego (Ferreyra et al. 2007). Lo anterior se evidencia en que en a.m. la $\mathrm{T}_{\mathrm{h}}$ de los tratamientos CR y SR de ambos cultivos fue similar a la temperatura del aire hasta el día 5, posteriormente el día 6, la $\mathrm{T}_{\mathrm{h}}$ promedio de los tratamientos SR de ambos cultivos, fue en promedio 5 grados superior a la temperatura del aire.

Después del amanecer, la $\mathrm{ET}_{\mathrm{c}}$ incrementa gradualmente conforme al incremento de la radiación solar y la temperatura, y a la reducción de la humedad. En las primeras horas de la mañana, cuando las condiciones climáticas son óptimas, hay una buena relación entre el estado hídrico del sustrato, la planta y la atmósfera. La planta transpira sin estrés hídrico y la $\mathrm{T}_{\mathrm{h}}$ puede ser de 1 a $4^{\circ} \mathrm{C}$ menor que la temperatura ambiental (García-Petillo 2008).

Durante las horas alrededor del m.d., bajo condiciones críticas de alta radiación solar (1200 $\left.\mu \mathrm{mol} \mathrm{m} \mathrm{m}^{-2} \cdot \mathrm{s}^{-1}\right)$, temperatura $\left(35^{\circ} \mathrm{C}\right)$ y baja humedad relativa (64\%), la transpiración se eleva al máximo excediendo la capacidad de absorción de agua del cultivo. Ferreyra et al. (2007) demostraron que bajo dichas condiciones climáticas el déficit de transpiración es cubierto por el agua del tejido, lo cual reduce el $\psi_{\mathrm{h}}$ y ocasiona el cierre de estomas. Por tales razones, Galvez et al. (2011) recomiendan que para efectos de manejo del riego, el $\psi_{\mathrm{h}}$ es útil cuando existe la máxima demanda de agua.

Respecto al comportamiento mostrado en $\psi_{\mathrm{h}}$, en ambos cultivos se presentaron diferencias $(\mathrm{p}<0,05)$ al m.d. del día 2, donde los valores de $\psi_{\mathrm{h}}$ en tomate fueron -0,84 MPa en el tratamiento CR y $-1,24 \mathrm{MPa}$ en SR. En tomate bien regado García et al. (2007) reportaron valores de $\psi_{\mathrm{h}}$ de $<-0,6 \mathrm{MPa}$ y de -1,25 MPa bajo estrés hídrico, mientras que Ben-Asher et al. (1992) reportaron mínimo estrés hídrico cuando el $\psi_{\mathrm{h}}$ fue $-1 \mathrm{MPa}$ en cultivo de tomate bien regado. Por otra parte, Duniway (1971), citado por Zegbe y Behboudian (2006), estableció un umbral crítico en tomate de <-1 MPa, que teóricamente induciría al cierre estomático. En chile dulce CR los valores de $\psi_{\mathrm{h}}(-0,98 \mathrm{MPa})$ fueron similares a los valores reportados por Thompson et al. (2007a) y Dorji y Behboudian (2005); mientras que en chile SR (-1.3 MPa) fueron muy similares a los reportados por García et al. (2007) en cultivos sin riego (-1,24 MPa).

En este estudio, al m.d. la temperatura promedio del aire $\left(32,9^{\circ} \mathrm{C}\right)$ y la $\mathrm{T}_{\mathrm{h}}$ de ambos cultivos SR fueron muy similares. En los cultivos $\mathrm{CR}$ al m.d., las $\mathrm{T}_{\mathrm{h}}$ en tomate $\left(26,3^{\circ} \mathrm{C}\right)$ y en chile dulce $\left(25,5^{\circ} \mathrm{C}\right)$ fueron $6,5^{\circ} \mathrm{C}$ y $7,3^{\circ} \mathrm{C}$ inferiores a la temperatura del aire, respectivamente. Cuando la planta cierra estomas se reduce la transpiración, lo cual afecta la capacidad de enfriamiento evaporativo del dosel vegetal. En dicha condición, la $\mathrm{T}_{\mathrm{h}}$ asciende y puede alcanzar de 4 a $6^{\circ} \mathrm{C}$ más que la temperatura del aire (López et al. 2009).

Diversos estudios han demostrado que el estrés hídrico a nivel de planta puede presentarse mucho antes de haber alcanzado un nivel de agotamiento de la $\theta_{\text {vs }}$ preestablecido (Thompson et al. 2007a). Por tal razón, el umbral de humedad en la fibra de coco, en el cual debe iniciar el riego, debe establecerse durante las primeras horas de la mañana cuando no ha sobrepasado $38 \%$ de $\theta_{\mathrm{vs}}$ a una tensión de succión matricial de $-5 \mathrm{kPa}$, definido según Ansorena (1994) como el umbral límite del agua fácilmente disponible en la fibra de coco (Figura 1). Además, en el rango de $56 \%$ a $38 \%$ de $\theta_{\mathrm{vs}}, \operatorname{los} \psi_{\mathrm{h}}$ fueron aproximadamente -0,5 MPa y las $\mathrm{T}_{\mathrm{h}}$ fueron similares a la temperatura del aire.

\section{LITERATURA CITADA}

Abad, M; Noguera, P; Puchades, R; Maquieira, A; Noguera, V. 2002. Physico-chemical and chemical properties of some coconut coir dusts for use as a peat substitute for containerised ornamental plants. Bioresource Technology 82:241-245.

Ali, MH. 2010. Crop Water Requirement and Irrigation Scheduling. En Fundamentals of irrigation and On-farm water management (1):399-452. 
Allen, RG; Pereira, LS; Raes, D; Smith, M. 2006. Evapotranspiración del cultivo Guías para la determinación de los requerimientos de agua de los cultivos. Estudio FAO-56. Riego y Drenaje. Roma, Italia. 323 p.

Ansorena, MJ. 1994. Sustratos. Propiedades y caracterización. Madrid, España. Ed. Mundi Prensa. 167 p.

Arguedas, F; Lea-Cox, J; Méndez, CH. 2006. Calibration of ECH20 Probe Sensors to Accurately Monitor Water Status of Traditional and Alternative Substrates for Container Production. SNA Research Conference: Water Management 51:501-505.

Ben-Asher, J; Phene, CJ; Kinarti, A. 1992. Canopy temperature to assess daily evapotranspiration and management of high frequency drip irrigation systems. Agricultural Water Management 22(4):379390 .

Capraro, F; Tosetti, S: Vita, F; Patiño, D; Schugurensky, C; Fullana, R. 2008. Jornadas de Inf. Ind. Agroinformática. Sistema de monitoreo continuo de la humedad en suelo para el control de riego en un olivar (Olea europaea L.) empleando LabVIEW. $37^{\circ}$. San Juan Argentina. p. 28-42.

Chen, J; Kang, S; Du, T; Qiu, R; Guo, P; Chen, R. 2013. Quantitative response of greenhouse tomato yield and quality to water deficit at different growth stages. Agricultural Water Management 129:152162.

Christiansen, JE. 1942. Irrigation by Sprinkling. California Agriculture Experiment Station Bulletin No. 670 . 126 p. Consultado 20 set. 2017. Disponible en https:// archive.org/details/irrigationbyspri670chri

Coates, RW; Delwiche, MJ; Broad, A; Holler, M. 2013. Wireless sensor network with irrigation valve control. Computers and Electronics in Agriculture. Computers and Electronics in Agriculture 96:13-22.

Corell, M; Girón, IF; Galindo, A; Torrecillas, A; TorresSánchez, R. 2014. Using band dendrometers in irrigation scheduling Influence of the location inside the tree and comparison with point dendrometer. Agricultural Water Management 142:29-37.

Dorji, K; Behboudian, MH. 2005. Water relations, growth , yield, and fruit quality of hot pepper under deficit irrigation and partial rootzone drying. Scientia Horticulturae 104:137-149.

Elliott, J; Deryng, D; Müller, C; Frieler, K; Konzmann, M; Gerten, D; Glotter, M; Flörkeg, M; Wadah, I; Besta, N; Eisnerg, S; Feketei, BM; Folberthj, C; Fostera, I; Goslingk, SN; Haddelandl, I; Khabarovm, N; Ludwign, F; Masakio, Y; Olinp, S; Rosenzweigc, C; Ruanec, AC; Satohr, Y; Schmids, E; Stacket, T; Tangu, Q; Wisser, D. 2014. Constraints and potentials of future irrigation water availability on agricultural production under climate change. Proceedings of the National Academy of Sciences 111(9):3239-3244.
Ferreyra, R; Selles, G; Maldonado, P; Celedón, J; Gil, P. 2007. Efecto del clima, de las características de la hoja y de la metodología de medición en el potencial hídrico xilemático en palto (Persea americana Mill.). Agricultura técnica 67(2):182-188.

Gallardo, M; Soto, F; Giménez, C; Martínez-Gaitán, C; Thompson, RB. 2011. Simulación de la productividad y dinámica del agua y $\mathrm{N}$ en cultivo de pimiento bajo invernadero con EU-ROTATE_N. Avances en nuevas estrategias de fertilización 56:17-22.

Galvez, PR; Callejas, RR; Reginato, MG. 2011. Comparación de la cámara de presión tipo Scholander modelo Pump-up respecto a la cámara de presión tradicional en vides de mesa. Idesia 29(2):175-179.

García-Petillo,M.2008. Manejo del riego: uso de instrumentos de medición de agua del suelo y del estado hídrico de los cultivos, presentación de casos de estudio incluso en riego deficitario. Jornadas sobre "Ambiente y Riegos : Modernización y Ambientalidad”. 2008. La Antigua, Guatemala. 20 p.

García, AL; Marcelis, L; Nicolas, N; Martínez, V. 2007. Moderate water stress affects tomato leaf water relations in dependence on the nitrogen supply. Biologia plantarum 51(4):707-712.

Goumopoulos, C; Flynn, BO; Kameas, A. 2014. Automated zone-specific irrigation with wireless sensor/actuator network and adaptable decision support. Computers and electronics in agriculture 105:20-33.

Incrocci, L; Marzialetti, P; Incrocci, G; Di, A; Balendonck, J; Bibbiani, C; Spagnol, S; Pardossi, A. 2014. Substrate water status and evapotranspiration irrigation scheduling in heterogenous container nursery crops. Agricultural Water Management 131:30-40.

Kirda, C; Cetin, M; Dasgan, Y; Topcu, S; Kaman, H; Ekici, B; Derici, MR; Ozguven, AI. 2004. Yield response of greenhouse grown tomato to partial root drying and conventional deficit irrigation. Agricultural Water Management 69:191-201.

Kuşçu, H; Turhan, A: Demir, A. 2014. The response of processing tomato to deficit irrigation at various phenological stages in a sub-humid environment. Agricultural Water Management 133:92-103.

López, LR; Ramírez, A; Peña, V; Alberto, M; Cruz, L. 2009. Índice de estrés hídrico como un indicador del momento de riego en cultivos agrícolas. Agric. Téc. Méx. 35(1):92-106.

Navarro-Hellin, H; Torres-Sánchez, R; Soto-Valles, F; Albaladejo-Pérez, C; López-Riquelme, JA; DomingoMiguel, R. 2015. A wireless sensors architecture for efficient irrigation water management. Agricultural Water Management 151:64-74.

Pardossi, A; Incrocci, L. 2011. Traditional and New Approaches to Irrigation Scheduling in Vegetable Crops. Hortechnology 21(3):309-313.

Pardossi, A; Incrocci, L; Incrocci, G; Malorgio, F; Battista, P; Bacci, L; Rapi, B; Marzialetti, P; Hemming, 
J; Balendonck, J. 2009. Root Zone Sensors for Irrigation Management in Intensive Agriculture. Sensors 9:2809-2835.

Pilatti, MA; Orellana, JD; Imhoff, SC; Pires, DA. 2012. Review of the critical limits of the optimal hydric interval. Antecedentes del IHO : agua útil y Non Limiting Water Range (NLWR) agua comenzando a afectarse el crecimiento del cultivo otras propiedades físicas sobre el crecimiento de los cul- no reduzca. Ciencia del Suelo 30(1):9-21.

Rhie, YH; Kim, J. 2017. Changes in Physical Properties of Various Coir Dust and Perlite Mixes and Their Capacitance Sensor Volumetric Water Content Calibrations. Hort Science 52(1):162-166.

San Martín, JP; Acevedo, E. 2001. Temperatura de canopia, CWSI y rendimiento en genotipos de trigo. Laboratorio de Relación Suelo-Agua-Planta. Universidad de Chile, Santiago de Chile. 61 p.

Soto, F; Gallardo, M; Giménez, C; Peña-Fleitas, T; Thompson, RB. 2014. Simulation of tomato growth, water and $\mathrm{N}$ dynamics using the EU-Rotate_N model in Mediterranean greenhouses with drip irrigation and fertigation. Agricultural Water Management 132:46-59.

Starr, JL; Paltineanu, IC. 1998. Soil water dynamics using multisensor capacitance probes in nontraffic interrows of corn. Soil Sci Soc Amer J. 62(1):114-122.

Steel, RGD; Torrie, JH. 1980. Principles and Procedures of Statistics. Second edition New York. McGrawHill. 633 p.

Thompson, RB; Gallardo, M; Valdez, LC; Fernández, MD. 2007a. Determination of lower limits for irrigation management using in situ assessments of apparent crop water uptake made with volumetric soil water content sensors. Agricultural Water Management 92:13-28.
Thompson, RB; Gallardo, M; Valdez, LC; Fernández, MD. 2007b. Using plant water status to define threshold values for irrigation management of vegetable crops using soil moisture sensors. Agricultural Water Management 88:147-158.

Tilman, D; Cassman, KG; Matson, PA; Naylor, R; Polasky, S. 2002. Agricultural sustainability and intensive production practices. Nature 418(6898):671-677.

Van Der Westhuizen, RJ. 2009. Irrigation scheduling of tomatoes (Lycopersicon esculentum Mill.) and cucumbers (Cucumis sativus L.) grown hydroponically in coir. Disertation Ph.D. Stellenbosch, South Africa. Stellenbosch University. $139 \mathrm{p}$.

Van Iersel, MW; Dove, S; Kang, JG; Burnett, SE. 2010 Growth and water use of petunia as affected by substrate water content and daily light integral. Hort Science 45(2):277-282.

Vence, LB; Valenzuela, OR; Svartz, HA; Conti, ME. 2013. Elección del sustrato y manejo del riego utilizando como herramienta las curvas de retención de agua. Ciencia del Suelo 31(2):153-164.

Vellidis, G; Tucker, M; Perry, C; Kvien, C; Bednarz, C. 2008. A real-time wireless smart sensor array for scheduling irrigation. Computers and Electronics in Agriculture 61:44-50.

WWAP (United Nations World Water Assessment Programme). 2016. The United Nations World Water Development Report 2016: Water and Jobs. Paris, Francia. UNESCO (Organización de las Naciones Unidas para la Educación).167 p.

Zar, JH. 1999. Biostatistical Analysis. Cuarta edición. New Jersey. Prentice Hall. 663 p. Appendix A.

Zegbe, JA; Behboudian, MH. 2006. Respuesta del tomate para proceso al riego parcial de la raíz. TERRA Latinoamericana 25:61-67.

Todos los derechos reservados. Universidad de Costa Rica. Este artículo se encuentra licenciado con Creative Commons Reconocimiento-NoComercial-SinObraDerivada 3.0 Costa Rica. Para mayor información escribir a rac.cia@ucr.ac.cr 